\title{
QUALIDADE DA ÁGUA DO RIO CONRADO LOCALIZADO NA BACIA HIDROGRÁFICA DO RIO PATO BRANCO - SUDOESTE DO ESTADO DO PARANÁ
}

\section{Water quality of Conrado river located in Pato Branco river basin - southwestern of the Paraná State}

\author{
William Cesar Pollonio MACHADO1 \\ Valter Antonio BECEGATO² \\ André Virmond Lima BITTENCOURT ${ }^{3}$ \\ João Batista Pereira CABRAL ${ }^{4}$
}

\section{RESUMO}

A bacia hidrográfica do rio Pato Branco, manancial de abastecimento público da cidade de Pato Branco localizada no Sudoeste do Estado do Paraná com uma população de 70.000 habitantes, foi monitorada pelo período de dois ciclos hidrológicos, totalizando 22 campanhas em duas estações de monitoramento: a estação Conrado montante (RC01) e a Conrado jusante (RC02), locadas no rio Conrado. As principais características da bacia estudada são: área de $29,69 \mathrm{~km}^{2}$, comprimento 9.300 metros, desnível 170 metros, declividade $18,62 \mathrm{~m} / \mathrm{km}$ e tempo de concentração 102 minutos. Foram analisadas variáveis físico-químicas, cujas amostras foram coletadas respeitando um período mínimo de três dias sem chuvas. O conjunto de dados referente a sete variáveis para as estações de monitoramento RC01 e RC02 apresentaram respectivamente, as seguintes médias: variáveis que caracterizam a matéria orgânica: $\mathrm{DBO}_{5}(5,2-4,31)$ e DQO $(11,04$ - 11,2) e as que caracterizam os processos de eutrofização: $\mathrm{N}$ amoniacal $(0,16-0,09)$; nitrato $(0,67-0,78)$; nitrito $(0,06$ $-0,029) ; \mathrm{N}$ total $(0,48-0,33)$ e fósforo total $(0,13-0,07)$. Os menores e maiores coeficientes de variação dos dados a montante e a jusante respectivamente, foram observados nas variáveis: nitrato e nitrito (46,1 e $166 \%)$; fósforo e nitrito (56,8 e $229,6 \%)$.

Palavras-chave: qualidade da água; bacia hidrográfica; rio Conrado; Pato Branco.

\begin{abstract}
The Pato Branco river basin, which supplies water to the municipality of Pato Branco (70,000 inhabitants) in southwestern Paraná State, was monitored during two hydrologic cycles, totalizing 22 campaigns at two monitoring stations on the Conrado river: the Conrado station upstream (RC01) and the Conrado station downstream (RC02). The main characteristics of the basin are: area $-29.69 \mathrm{~km}^{2}$, length $-9,300 \mathrm{~m}$, height difference $-170 \mathrm{~m}$, declivity $-18.62 \mathrm{~m} / \mathrm{km}$, and concentration time - $102 \mathrm{~min}$. Physico-chemical variables were analyzed, using samples collected after a minimum three-day period without rain. The data set comprising seven variables yielded the following averages respectively for monitoring stations RC01 and RC02: variables that characterize organic matter: $\mathrm{BOD}_{5}(5.2-4.31)$ and $\mathrm{COD}(11.04-11.2)$, and variables that characterize eutrophication processes: ammoniacal $\mathrm{N}(0.16-$ $0.09)$; nitrate $(0.67-0.78)$; nitrite $(0.06-0.029)$; total $\mathrm{N}(0.48$ $-0.33)$, and total phosphorous $(0.13-0.07)$. The lowest and the highest variation coefficients for data downstream and upstream correspond to: nitrate and nitrite (46.1 and 166\%); phosphorous and nitrite (56.8 and $229.6 \%$ ).
\end{abstract}

Keywords: water quality; river basin; Conrado river; Pato Branco.

\footnotetext{
1 Professor Doutor - UFTPR - Unidade Pato Branco-PR e-mail - wcpm@mail.crea-pr.org.br

2 Professor Doutor - UDESC/Lages-SC - e-mail - becegato@cav.udesc.br

3 Professor Doutor - UFPR - e-mail - andre@ufpr.br

4 Professor Doutor - UFGO - e-mail - Jbcabral2000@yahoo.com.br
} 
MACHADO, W. C. P. et al. Qualidade da água do Rio Conrado localizado na...

\section{INTRODUÇÃO}

O presente trabalho faz parte do projeto PNMA (Projeto Nacional de Monitoramento Ambiental - setor de suinocultura) promovido pelo Ministério do Meio Ambiente e teve como objetivo principal o monitoramento da qualidade da água do rio Conrado, cujas amostras para análises foram colhidas a montante e a jusante durante um período de dois ciclos hidrológicos, dando ênfase à avaliação do aporte de matéria orgânica, inorgânica e dos compostos nitrogenados em período de escoamento dos corpos hídricos aproximando do fluxo de base.

A inserção da bacia hidrográfica do rio Pato Branco dentro do referido projeto, deveu-se à atividade de suinocultura considerada de subsistência, além de outros usos. A partir de novembro de 2003 , foram iniciadas as avaliações da qualidade da água nas sub-bacias dos rios Conrado e Pinheiro inseridas na Bacia do Rio Pato Branco, visando buscar uma forma de interpretação da situação da qualidade das águas superficiais para as bacias rurais, procurando estender este conceito para outras bacias com as mesmas características, o que seria próprio para a região Sudoeste do Estado do Paraná (MACHADO et al., 2006).

A água tem estado nas grandes discussões ambientais mundiais como um insumo de tal importância para alguns países, que passou a ser tema de constante preocupação com realação o seu uso tanto no aspecto industrial como para consumo humano. O conjunto das atividades humanas, cada vez mais diversificado, associado ao crescimento demográfico, vem exigindo maior atenção às necessidades de uso da água para as mais diversas finalidades (MANCUSO et al. 2003).

Há ainda que se considerar que o meio ambiente se apresenta sob diversas dimensões, formas, significados e evolui através do tempo. No entanto, quando a consideração para análise envolve a dimensão das ações antrópicas e seu entorno, encontramos um ambiente social significando as relações de indivíduos dentro da mesma comunidade, e a partir destas relações e das necessidades das comunidades, é gerado um maior interesse na exploração dos recursos naturais, cada vez mais sustentado por processos tecnológicos modernos, numa velocidade de extração mais rápida e utilizando uma maior quantidade de energia. Esta análise conduz para uma maior exploração dos recursos naturais a qual sem o devido gerenciamento produz degradação, podendo levar a efeitos irreversíveis nos ciclos naturais e entre esses o ciclo hidrológico.

Neste contexto, surge a necessidade de se incrementar o conhecimento sobre as bacias hidrográficas, considerando o seu uso atual bem como a sua capacidade máxima de uso, sob o ponto de vista de todas as atividades existentes na sua área de contribuição, visando um controle em termos de qualidade da água, que possa ser aplicado para bacias similares.

\section{MATERIAL E MÉTODOS}

Localizada no Terceiro Planalto Paranaense, entre as latitudes extremo norte: $\mathrm{E}=341769 \mathrm{~N}=7091983$, extremo oeste: $E=331010 \mathrm{~N}=7087501$, extremo sul: $E=340438 \mathrm{~N}=7077212$, extremo leste: $E=347288$ $\mathrm{N}=7079699$, a bacia hidrográfica do Rio Pato Branco possui uma área de contribuição de $130,43 \mathrm{Km}^{2}$, sub bacia do Rio Chopin inserida na Bacia hidrográfica do Rio Iguaçu. A área apresenta intensa ação antrópica devido a sua localização estratégica para o desenvolvimento inicial do município e por se tratar do ponto de chegada dos colonizadores nas décadas de 1950, 1960 e 1970, bem como ser o caminho de passagem dos mesmos que imigraram dos Estados do Rio Grande do Sul e de Santa Catarina.

O projeto de monitoramento quali-quantitativo das águas na bacia hidrográfica do rio Conrado teve suas atividades concentradas no período de outubro/2003 a julho/2005, com coletas manuais a montante e a jusante, cujas estações de monitoramento foram denominadas de RC01 (a montante) e RC02 (a jusante) do rio Conrado, totalizando 22 campanhas com frequência mensal, respeitando um período na data de coleta de no mínimo três dias sem chuva.

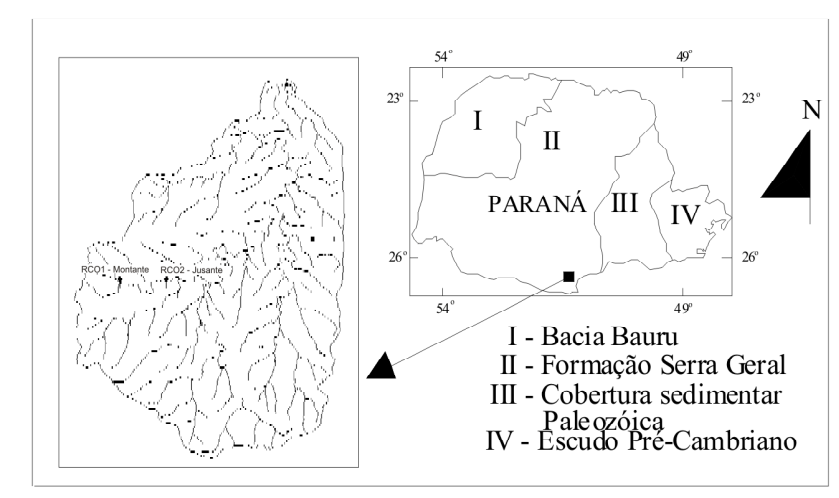

FIGURA 1 - LOCALIZAÇÃO DA BACIA HIDROGRÁFICA DO RIO PATO BRANCO E AS RESPECTIVAS ESTAÇÕES DE MONITORAMENTO PARA COLETAS DAS AMOSTRAS DE ÁGUA 
MACHADO, W. C. P. et al. Qualidade da água do Rio Conrado localizado na...

Tal procedimento objetivou verificar a tendência do escoamento de base do referido rio, bem como procurar estabelecer uma caracterização das águas próxima da sua constituição natural. As amostras foram enviadas para o laboratório do Instituto Ambiental do Paraná (IAP) em Curitiba e da UTFPR (Universidade Tecnológica Federal do Paraná - Campus de Pato Branco), levandose em consideração todos os requisitos para a coleta (recipiente de inox), preservação da amostra no campo com gelo e transporte no menor tempo possível.

\section{RESULTADOS E DISCUSSÃO}

Nas Tabelas 1 e 2 encontram-se os resultados dos parâmetros analisados nas amostras de água com os valores das sete variáveis monitoradas referentes a
22 campanhas realizadas a jusante e a montante do Rio Conrado, bem como suas respectivas estatísticas.

\section{VARIÁVEIS QUE CARACTERIZAM A MATÉRIA ORGÂNICA}

\section{DEMANDA BIOQUÍMICA DE OXIGÊNIO (DBO)}

Conceitualmente a DBO indica a quantidade de oxigênio necessária para oxidar a matéria orgânica mediante processos bioquímicos aeróbicos por um período de incubação é de cinco dias a $20^{\circ} \mathrm{C}$ para formas inorgânicas estabilizadas. Esta determinação é conhecida como $\mathrm{DBO}_{5}$ e informa indiretamente se os corpos

\begin{tabular}{|c|c|c|c|c|c|c|c|}
\hline & \multicolumn{5}{|c|}{ Fósforo } & \multirow{2}{*}{$\begin{array}{c}\text { Nitrogênio } \\
\text { Amoniacal } \\
\text { mg/L N }\end{array}$} & \multirow{2}{*}{$\begin{array}{c}\text { Nitrogênio } \\
\text { Total } \\
\text { mg/L N }\end{array}$} \\
\hline $\begin{array}{c}\text { Data da } \\
\text { coleta }\end{array}$ & $\begin{array}{c}\mathrm{DBO}_{5} \\
\mathrm{mg} / \mathrm{L} \mathrm{O}_{2}\end{array}$ & $\begin{array}{c}\mathrm{DQO} \\
\mathrm{mg} / \mathrm{L} \mathrm{O}_{2}\end{array}$ & $\begin{array}{l}\text { Total } \\
\mathrm{mg} / \mathrm{L}\end{array}$ & $\begin{array}{l}\text { Nitrato } \\
\mathrm{mg} / \mathrm{L} \mathrm{N}\end{array}$ & $\begin{array}{l}\text { Nitrito } \\
\mathrm{mg} / \mathrm{L} \mathrm{N}\end{array}$ & & \\
\hline $04 / 11 / 03$ & 6 & 9 & 0,09 & 0,93 & 0,46 & 0,16 & 0,66 \\
\hline $09 / 12 / 03$ & 28 & 31 & 0,15 & 1,08 & 0,11 & 0,42 & 0,63 \\
\hline $05 / 01 / 04$ & 4 & 7 & 0,526 & 1,08 & 0,062 & 0,34 & 0,52 \\
\hline $11 / 02 / 04$ & 4 & 5 & 0,09 & 1,06 & 0,012 & 0,16 & 1,16 \\
\hline $25 / 02 / 04$ & 7 & 12 & 0,16 & 0,1 & 0,039 & 0,04 & 0,234 \\
\hline $23 / 03 / 04$ & 4 & 12 & 0,09 & 0,67 & 0,005 & 0,005 & 0,014 \\
\hline $27 / 04 / 04$ & 2 & 3 & 0,09 & 0,74 & 0,17 & 0,17 & 0,973 \\
\hline $26 / 05 / 04$ & 4 & 11 & 0,18 & 0,71 & 0,01 & 0,16 & 0,52 \\
\hline $01 / 06 / 04$ & 2 & 5 & 0,03 & 0,023 & 0,001 & 0,03 & 0,16 \\
\hline $30 / 06 / 04$ & 7 & 21 & 0,09 & 0,79 & 0,021 & 0,06 & 0,31 \\
\hline $27 / 07 / 04$ & 6 & 10 & 0,16 & 0,76 & 0,015 & 0,04 & 0,17 \\
\hline $24 / 08 / 04$ & 2 & 5 & 0,27 & 0,73 & 0,036 & 0,33 & 0,54 \\
\hline 05/10/04 & 2 & 13 & 0,14 & 0,91 & 0,053 & 0,08 & 0,32 \\
\hline $28 / 10 / 04$ & 2 & 12 & 0,07 & 0,61 & 0,011 & 0,39 & 0,32 \\
\hline $01 / 12 / 04$ & 2 & 14 & 0,12 & 0,62 & 0,006 & 0,041 & 0,2 \\
\hline $14 / 12 / 04$ & 19 & 20 & 0,14 & 0,28 & 0,021 & 0,24 & 0,52 \\
\hline $01 / 02 / 05$ & 2 & 14 & 0,05 & 0,31 & 0,016 & 0,07 & 0,32 \\
\hline $21 / 02 / 05$ & 5 & 21 & 0,13 & 0,48 & 0,073 & 0,54 & 1,13 \\
\hline $29 / 03 / 05$ & 2 & 8 & 0,2 & 1,1 & 0,066 & 0,21 & 0,58 \\
\hline $03 / 05 / 05$ & 2 & 6 & 0,061 & 0,54 & 0,003 & 0,02 & 0,66 \\
\hline $30 / 05 / 05$ & 1 & 2 & 0,041 & 0,64 & 0,013 & 0,033 & 0,16 \\
\hline $27 / 07 / 05$ & 1 & 2 & 0,08 & 0,6 & 0,172 & 0,038 & 0,58 \\
\hline \multicolumn{8}{|c|}{ Estatísticas } \\
\hline Mínimo & 1 & 2 & 0,03 & 0,02 & 0,001 & 0,005 & 0,01 \\
\hline Máximo & 28 & 31 & 0,526 & 1,1 & 0,46 & 0,54 & 1,16 \\
\hline Média & 5,2 & 11,04 & 0,13 & 0,67 & 0,06 & 0,16 & 0,48 \\
\hline Mediana & 2 & 10,5 & 0,10 & 0,71 & 0,02 & 0,07 & 0,52 \\
\hline Desvio padrão & 6,4 & 7,22 & 0,104 & 0,31 & 0,10 & 0,16 & 0,31 \\
\hline $\begin{array}{c}\text { Coeficiente de } \\
\text { variação }(\%)\end{array}$ & 123 & 65,4 & 80 & 46,1 & 166 & 99 & 63,6 \\
\hline Assimetria & 2,85 & 1,08 & 2,72 & $-0,49$ & 3,10 & 1,0 & 0,78 \\
\hline Curtose & 8,44 & 1,3 & 9,41 & $-0,25$ & 10,98 & $-0,13$ & 0,23 \\
\hline
\end{tabular}

TABELA 1 - RESULTADOS DAS ANÁLISES DA QUALIDADE DA ÁGUA E RESUMO ESTATÍSTICO A MONTANTE DA ESTAÇÃO DE MONITORAMENTO RC01 
MACHADO, W. C. P. et al. Qualidade da água do Rio Conrado localizado na...

\begin{tabular}{|c|c|c|c|c|c|c|c|}
\hline & \multicolumn{5}{|c|}{ Fósforo } & \multirow{2}{*}{$\begin{array}{c}\text { Nitrogênio } \\
\text { Amoniacal } \\
\text { mg/L N }\end{array}$} & \multirow{2}{*}{$\begin{array}{c}\text { Nitrogênio } \\
\text { Total } \\
\text { mg/L N }\end{array}$} \\
\hline $\begin{array}{c}\text { Data da } \\
\text { coleta }\end{array}$ & $\begin{array}{c}\mathrm{DBO}_{5} \\
\mathrm{mg} / \mathrm{L} \mathrm{O}_{2}\end{array}$ & $\begin{array}{c}\mathrm{DQO} \\
\mathrm{mg} / \mathrm{L} \mathrm{O}_{2}\end{array}$ & $\begin{array}{l}\text { Total } \\
\text { mg/L }\end{array}$ & $\begin{array}{l}\text { Nitrato } \\
\mathrm{mg} / \mathrm{L} \mathrm{N}\end{array}$ & $\begin{array}{l}\text { Nitrito } \\
\mathrm{mg} / \mathrm{L} \mathrm{N}\end{array}$ & & \\
\hline $04 / 11 / 03$ & 14 & 20 & 0,09 & 1,24 & 0,03 & 0,08 & 0,45 \\
\hline $09 / 12 / 03$ & 30 & 33 & 0,125 & 0,93 & 0,23 & 0,59 & 0,77 \\
\hline $05 / 01 / 04$ & 4 & 9 & 0,11 & 2,17 & 0,23 & 0,38 & 0,87 \\
\hline $11 / 02 / 04$ & 2 & 3 & 0,09 & 1,04 & 0,011 & 0,07 & 1,23 \\
\hline $25 / 02 / 04$ & 4 & 9 & 0,09 & 0,24 & 0,01 & 0,08 & 0,07 \\
\hline $23 / 03 / 04$ & 3 & 12 & 0,09 & 0,003 & 0,02 & 0,005 & 0,329 \\
\hline $27 / 04 / 04$ & 1 & 2 & 0.09 & 0,311 & 0,02 & 0,17 & 0,0 \\
\hline $26 / 05 / 04$ & 3 & 7 & 0,11 & 0,82 & 0,01 & 0,11 & 0,35 \\
\hline $01 / 06 / 04$ & 6 & 10 & 0,03 & 0,019 & 0,001 & 0,027 & 0,068 \\
\hline $30 / 06 / 04$ & 2 & 18 & 0,047 & 0,98 & 0,005 & 0,03 & 0,19 \\
\hline $27 / 07 / 04$ & 2 & 9 & 0,046 & 0,96 & 0,007 & 0,02 & 0,23 \\
\hline $24 / 08 / 04$ & 2 & 14 & 0,005 & 0,53 & 0,004 & 0,07 & 0,19 \\
\hline $05 / 10 / 04$ & 2 & 13 & 0,044 & 0,96 & 0,003 & 0,043 & 0,32 \\
\hline $28 / 10 / 04$ & 2 & 12 & 0,091 & 0,94 & 0,005 & 0,032 & 0,23 \\
\hline $01 / 12 / 04$ & 4 & 16 & 0,16 & 0,59 & 0,034 & 0,047 & 0,54 \\
\hline $14 / 12 / 04$ & 2 & 15 & 0,064 & 0,28 & 0,0042 & 0,049 & 0,2 \\
\hline $01 / 02 / 05$ & 2 & 13 & 0,016 & 0,55 & 0,002 & 0,046 & 0,24 \\
\hline $21 / 02 / 05$ & 2 & 12 & 0,07 & 0,91 & 0,0036 & 0,034 & 0,22 \\
\hline $29 / 03 / 05$ & 3 & 3,5 & 0,073 & 1,1 & 0,0029 & 0,023 & 0,23 \\
\hline $03 / 05 / 05$ & 3 & 10 & 0,03 & 0,76 & 0,003 & 0,02 & 0,19 \\
\hline $30 / 05 / 05$ & 1 & 3 & 0,046 & 1,03 & 0,004 & 0,02 & 0,18 \\
\hline $27 / 07 / 05$ & 1 & 2 & 0,013 & 0,84 & 0,005 & 0,02 & 0,14 \\
\hline \multicolumn{8}{|c|}{ Estatísticas } \\
\hline Mínimo & 1 & 2 & 0,005 & 0,003 & 0,001 & 0,005 & 0 \\
\hline Máximo & 30 & 33 & 0,16 & 2,17 & 0,23 & 0,59 & 1,23 \\
\hline Média & 4,31 & 11,2 & 0,07 & 0,78 & 0,029 & 0,09 & 0,33 \\
\hline Mediana & 2 & 11 & 0,07 & 0,87 & 0,005 & 0,04 & 0,23 \\
\hline Desvio padrão & 6,3 & 7,07 & 0,039 & 0,47 & 0,06 & 0,14 & 0,29 \\
\hline \multirow{2}{*}{$\begin{array}{l}\text { Coeficiente de } \\
\text { variação (\%) }\end{array}$} & 146 & 63 & 55,7 & 60,1 & 229,6 & 156,4 & 90,8 \\
\hline & 3,61 & 1,25 & 0,26 & 0,76 & 2,9 & 2,92 & 1,85 \\
\hline $\begin{array}{l}\text { Assimetria } \\
\text { Curtose }\end{array}$ & 13,9 & 3,19 & $-0,23$ & 2,60 & 7,26 & 8,61 & 3,36 \\
\hline
\end{tabular}

TABELA 2 - RESULTADOS DAS ANÁLISES DA QUALIDADE DA ÁGUA E RESUMO ESTATÍSTICO A JUSANTE DA ESTAÇÃO DE MONITORAMENTO RC02

hídricos possuem boas condições de oxigenação e se está ocorrendo aporte de matéria orgânica nos corpos hídricos.

A Resolução n 357/05 do Conama (BRASIL, 2005), estabelece que o valor limite para a $\mathrm{DBO}_{5}$ é de $5 \mathrm{mg} / \mathrm{L}$ de $\mathrm{O}_{2}$. Analisando o conjunto de dados para esta variável, observa-se que ocorreram violações àquele limite em $27,7 \%$ dos valores para o conjunto de dados para a estação RC01 e 13,7\% para a estação RC02. No entanto, a média do conjunto de valores apresentou respectivamente $5,2 \mathrm{mg} / \mathrm{L}$ de $\mathrm{O}_{2}$ para a estação $\mathrm{RC01}$ e $4,31 \mathrm{mg} / \mathrm{L}$ de $\mathrm{O}_{2}$ para a estação RC02, semelhantes àqueles obtidos por Machado et al. (2006), cujos valores estão próximos ao limite de classificação para rio classe 2.

Segundo Von Sperling (1995) em ambientes naturais sem aporte de matéria orgânica, os valores para as concentrações da $\mathrm{DBO}_{5}$ enquadram-se no intervalo de 1 a $10 \mathrm{mg} / \mathrm{L}$ de $\mathrm{O}_{2}$. Considerando tal amplitude, apenas
$9,1 \%$ dos resultados do conjunto de dados para as duas estações podem ser caracterizados como oriundos de águas poluídas. Mcneely et al. (1979) e Perez (1992), relataram que águas superficiais com $\mathrm{DBO}_{5}$ inferiores a $4 \mathrm{mg} / \mathrm{L}$ de $\mathrm{O}_{2}$ são razoavelmente limpas, e aquelas com níveis maiores do que $10 \mathrm{mg} / \mathrm{L}$ de $\mathrm{O}_{2}$ são consideradas como poluídas em função do aporte de quantidades de material orgânico degradável. Pela definição do último autor, pode-se dizer que em $72,75 \%$ dos valores obtidos para a estação de monitoramento RC01 e em 86,4\% para a estação de monitoramento RC02, caracterizam as águas como limpas.

Conforme as estatísticas descritivas (Tabelas 1 e 2) observa-se que o coeficiente de variação $146 \%$ foi maior para a estação de monitoramento RC02 do que para a estação RC01 com 123\%. De acordo com a Figura 2, os dados seguem uma distribuição assimétrica positiva para ambas as estações. 

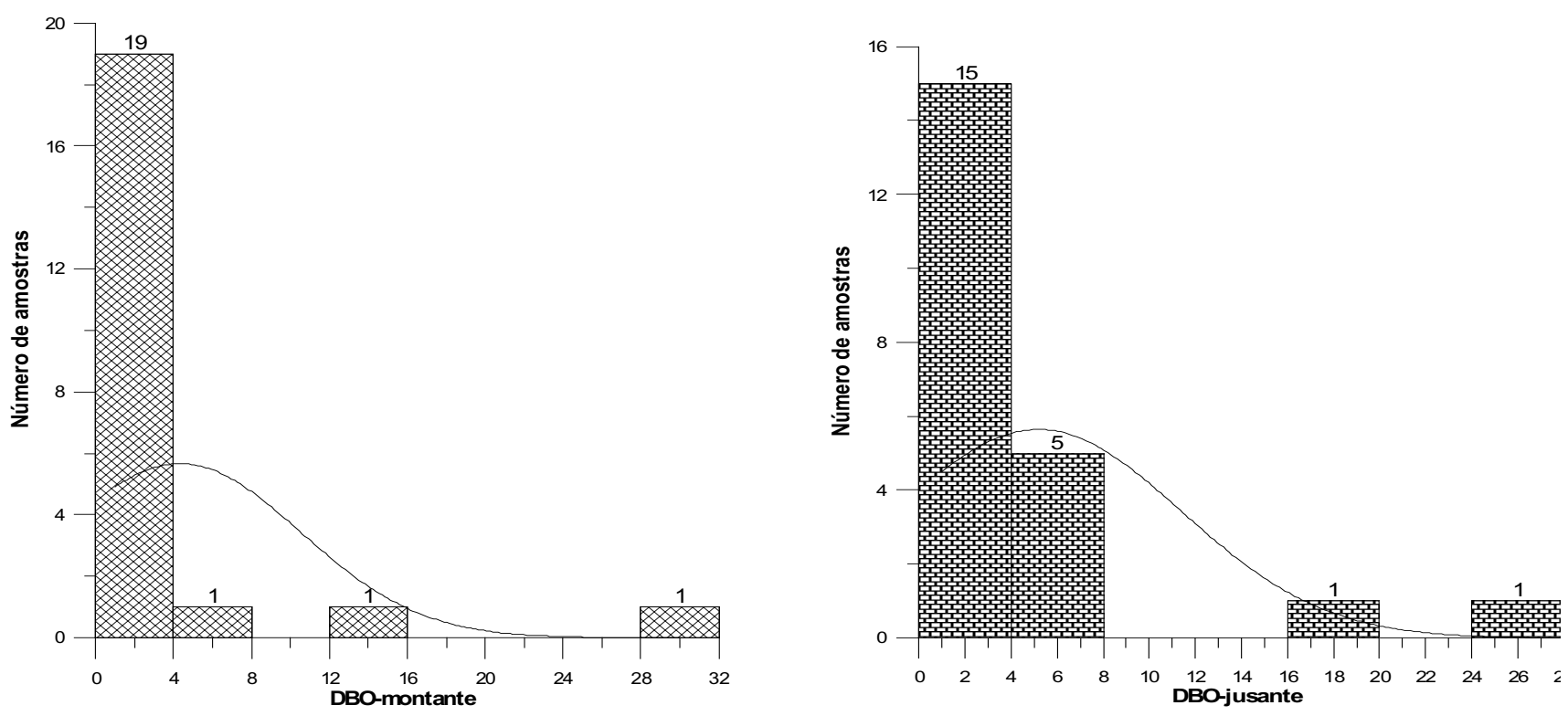

FIGURA 2 - HISTOGRAMAS DA DBO 5 PARA AS ESTAÇÕES DE MONITORAMENTO RC01 E RC02

O principal fator para o comportamento dos valores da DBO para as duas estações de monitoramento, deve-se a presença da criação intensiva de bovinos próximos ao divisor da bacia hidrográfica Iguaçu Uruguai, associado ao fato da utilização das áreas de preservação permanente para pastejo animal. Colabora ainda para aqueles valores a existência de duas industriais a montante da estação RC01, sendo um laticínio e um frigorífico para abate de aves.

\section{DEMANDA QUIIMICA DE OXIGÊNIO (DQO)}

A DQO é uma variável que determina o nível do oxigênio existente nos corpos hídricos. A oxidação da matéria orgânica se dá mediante um agente oxidante químico extremamente forte que oxida tanto a matéria orgânica como a inorgânica. Portando, o valor da DQO sempre será maior que a $\mathrm{DBO}_{5}$. No entanto as concentrações de $\mathrm{DQO}$ em águas superficiais podem atingir valores de até $20 \mathrm{mg} / \mathrm{L}$ de $\mathrm{O}_{2}$, sendo que neste intervalo as águas são consideradas menos poluídas
(CHAPMAN; KIMSTACH, 1996). Ainda segundo estes autores para valores de até $200 \mathrm{mg} / \mathrm{L}$ de $\mathrm{O}_{2}$ considera-se que as águas estão recebendo aporte de efluentes.

Embora a resolução $n^{\circ} 357 / 05$ do Conama (BRASIL, 2005) não faça referência a limites para esta variável e desta forma não foi possível avaliar se ocorreram violações, foi mantido como balizamento o limite estabelecido por Chapman e Kimstach (1996) que é de $20 \mathrm{mg} / \mathrm{L} \mathrm{de} \mathrm{O}_{2}$, muito embora tal valor possa ser considerado como sendo oriundo de efluente. Por esta avaliação foi observado que na campanha do dia 09/12/03 as duas estações apresentaram águas consideradas poluídas com valores de 28 e $30 \mathrm{mg} / \mathrm{L}$ de $\mathrm{O}_{2}$ respectivamente. Uma outra forma de avaliar o comportamento da DQO seria o aumento de concentração desta variável em um corpo hídrico, oriunda principalmente de efluentes industriais, em cuja composição se encontra materiais orgânicos redutores (MCNELLY et al.,1979). Conforme as estatísticas descritivas (tabelas 1 e 2), observa-se que o coeficiente de variação foi semelhante para ambas as estações $65,4 \%$ e $63 \%$ respectivamente, evidenciando um certo equilíbrio para a DQO. Os dados comportaram-se de forma assimétrica positiva para ambas as estações (figura 3 ). 

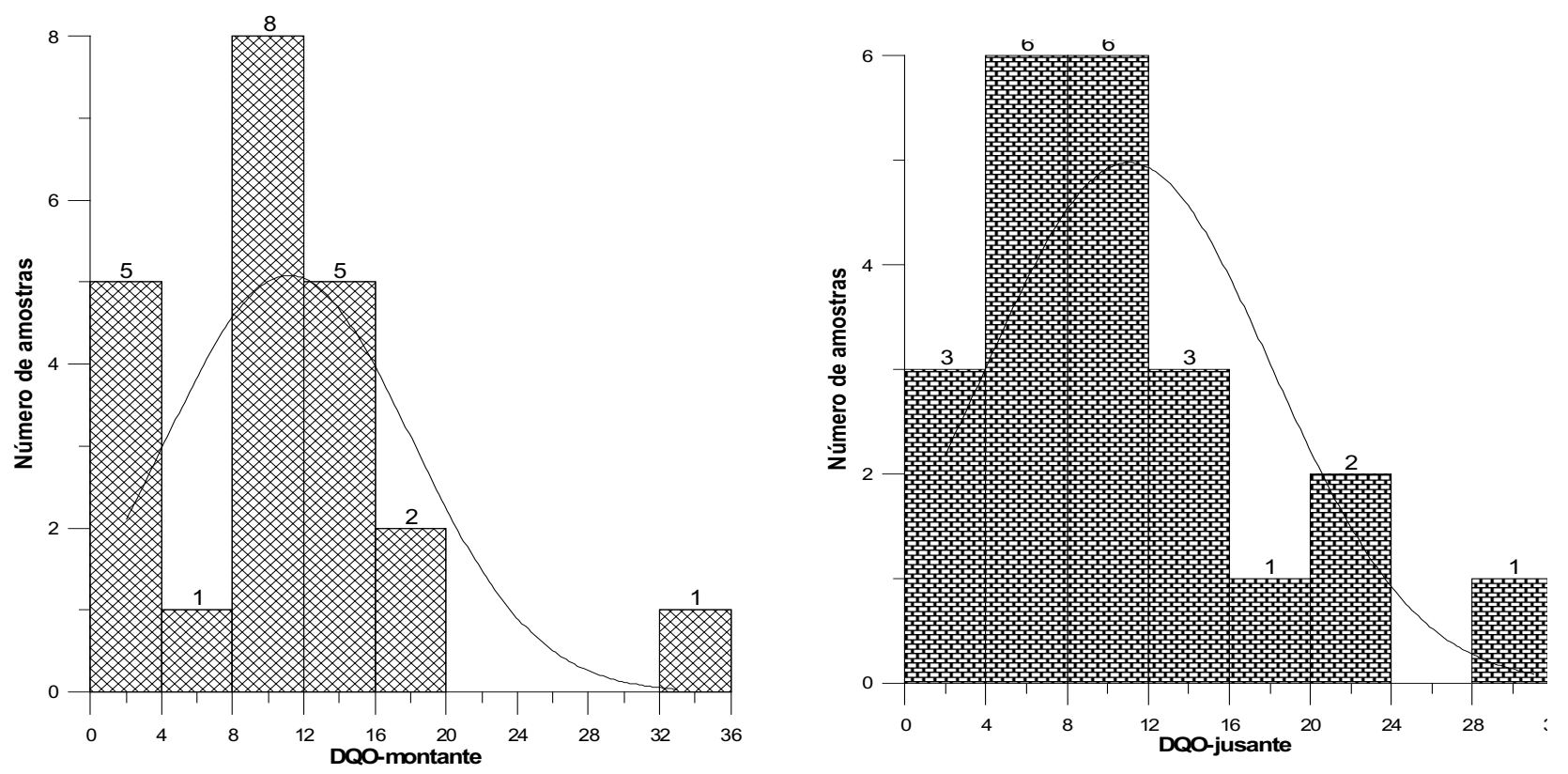

FIGURA 3 - HISTOGRAMAS DA DQO PARAAS ESTAÇÕES DE MONITORAMENTO RC01 E RC02

Analisando-se o conjunto de dados para as variáveis que representam a matéria orgânica $\left(\mathrm{DBO}_{5}\right.$ e a DQO) a maior dispersão dos valores ocorreu com a $\mathrm{DBO}_{5}$ (tabelas 1 e 2), cujas maiores variações foram encontradas nas estações de monitoramento situadas a jusante, enquanto que os valores médios foram maiores para as estações de montante. Pode-se inferir que o balanço inorgânico na bacia em estudo é mais equilibrado que o orgânico para as condições do presente estudo, ou pode ter havido maiores variações na qualidade das águas superficiais do rio pelo aporte de matéria orgânica.

\section{VARIÁVEIS QUE CARACTERIZAM O PROCESSO DE EUTROFIZAÇÃO}

\section{FÓSFORO TOTAL}

O aportamento da variável fósforo total no meio líquido pode ser de origem natural, oriundo da dissolução de rochas principalmente a apatita, carreamento de solo, decomposição da matéria orgânica e chuva, bem como advir de atividades antropogênicas pelo uso de fertilizantes químicos, agrotóxicos e efluentes, seja de origem industrial como laticínios, abatedouros e de esgotos, em que o elemento fósforo apresenta-se na forma de detergentes superfosfatados e matéria fecal.

Em geral pode ser encontrado na forma orgânica tanto solúvel (matéria orgânica solúvel dissolvida) como particulado (biomassa de microorganismo). Ainda pode estar na forma inorgânica solúvel (sais de fósforo) e inorgânica particulada (compostos minerais). Entre as formas apresentadas, a mais significativa é a inorgânica solúvel, $\mathrm{H}_{2} \mathrm{PO}_{4 \text { (aq.) }}^{-}(10 \%)$ e $\mathrm{HPO}_{4}^{-}$(aq.) $(90 \%)$ (FATMA, 1998).

Conforme as Tabelas 1 e 2 as maiores cargas apresentaram-se nos períodos de 09/12/03 e 05/01/04. Considerando-se que as amostras foram coletas dentro do intervalo de 3 dias sem chuvas e que o comportamento químico do fósforo seria de baixa mobilidadade, a explicação mais plausível pela alta carga seria o lançamento de efluentes das atividades industriais e a aplicação de dejetos suínos nos solos como adubo orgânico.

Quando são encontrados altos valores para o fósforo no meio líquido e dependendo das características do corpo receptor pode iniciar um processo de eutrofização. Em águas naturais as concentrações de fósforo apresentam-se na faixa de 0,01 a 0,05 mg/L de $\mathrm{P}$ (ESTEVES, 1998). Ainda de acordo com as referidas tabelas os valores médios do fósforo são de 0,13 e $0,07 \mathrm{mg} / \mathrm{L}$, respectivamente. Dvwk (1999) esclarece que em rios de correnteza baixa os teores críticos de 
fósforo para iniciar o processo de eutrofização estariam no intervalo de 0,1 a $0,2 \mathrm{mg} / \mathrm{L}$ de $\mathrm{P}$ e para rios de correnteza alta não deve ultrapassar o valor limite de $0,3 \mathrm{mg} / \mathrm{L}$ de $P$.

Agostinho e Gomes (1997), monitorando o reservatório de Segredo, informaram que a concentração média de fósforo total foi de $0,025 \mathrm{mg} / \mathrm{L}$ de $\mathrm{P}$ e que tal valor foi reduzido para $0,016 \mathrm{mg} / \mathrm{L}$ de P. Tal depleção dáse em função da absorção do fósforo pelo fitoplâncton e sua posterior sedimentação (THORNTON, 1990), bem como pela adsorção ao material particulado inorgânico e a precipitação do fósforo em compostos férricos (WETZEL, 1983). São fatores influentes na disponibilidade do fósforo a sua abundância relativa no ambiente e o tempo de residência da fração dissolvida. De forma geral os fosfatos rapidamente se complexam com cátions disponíveis no corpo hídrico, sendo os principais o ferro, o alumínio e o cálcio, formando complexos solúveis, quelatos e sais. Os principais fatores que governam estas formações e dissoluções destes compostos são: 0 $\mathrm{pH}$, concentração de fosfatos no corpo hídrico, potencial redox e as atividades da biota. Tais fatores removem o fosfato da coluna da água e reduzem a concentração de certos metais em função da precipitação dos compostos metalo-fosfóricos (CANADÁ, 1999).

A Resolução $n^{\circ} 375 / 05$ do Conama (BRASIL, 2005) estabelece que para rios de classe 2 o valor limite para o ambiente lótico é de $0,1 \mathrm{mg} / \mathrm{L}$ de P. Observa-se

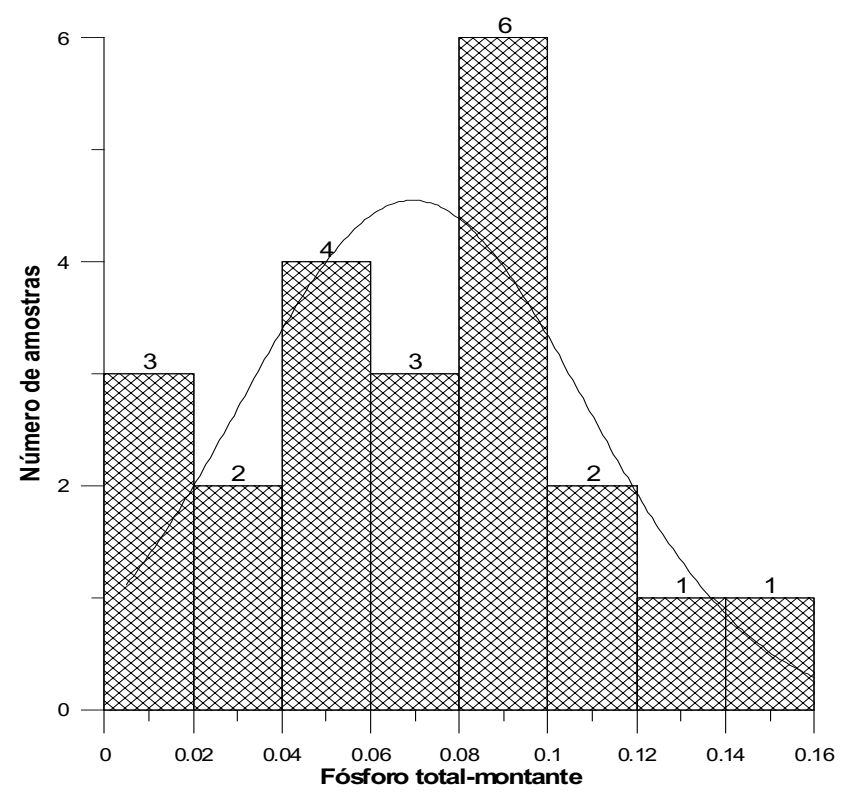

que $50 \%$ das amostras da estação de monitoramento RC01 estão acima do referido limite e que tais violações se distribuíram ao longo dos períodos das campanhas. No entanto para ambientes intermediários, considerando-se corpos hídricos que afluem para áreas de reservatórios em ambiente lêntico com tempo de residência entre 2 e 40 dias, o limite seria de $0,05 \mathrm{mg} / \mathrm{L}$ de P. O nível de violação atingiu $90,9 \%$ dos dados da estação automática RC01 e de 72,7\% para a estação RC02. Destaca-se que está sendo projetada uma série de usinas para o rio Chopin e que a bacia hidrográfica em estudo é contribuinte para os barramentos que serão edificados.

Como se está avaliando a tendência da qualidade das águas, é possível que o rio Conrado possua potencial para desenvolver processos de eutrofização na situação acima mencionada. Portanto, adota-se para análise uma comparação com os dois valores, mas devendo prevalecer no futuro o valor limite de 0,05 $\mathrm{mg} / \mathrm{L}$ de P. Cabe ainda ressaltar que foi a estação de monitoramento RC01 que apresentou os maiores valores e alterações. Um dos responsáveis pelo aumento da concentração de fósforo total pode ter sido o uso de fertilizantes agrícolas e agrotóxicos. Os dados são mais dispersos a montante com um coeficiente de variação de $80 \%$ comparativamente aos da jusante com $55,7 \%$ (Figura 4).

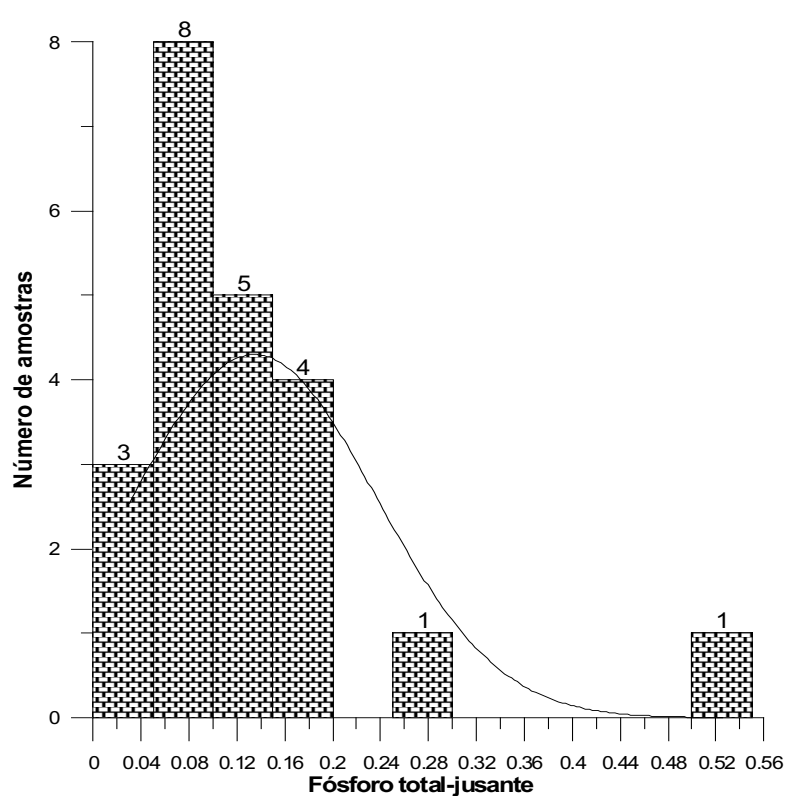

FIGURA 4 - HISTOGRAMAS DO FÓSFORO TOTAL PARAAS ESTAÇÕES DE MONITORAMENTO RC01 E RC02 


\section{COMPOSTOS NITROGENADOS}

A variável nitrito apresentou concentração média para a estação de monitoramento RC01 e RC02 de 0,06 e $0,029 \mathrm{mg} / \mathrm{L} \mathrm{N}$ respectivamente (Tabelas 1 e 2 ). Tais valores estão abaixo do que estabelece a Resolução do Conama $n^{\circ}$ 357/005 (BRASIL, 2005) que é de 1,0 $\mathrm{mg} / \mathrm{L} \mathrm{N}$. Em nenhuma das 22 campanhas, obteve-se valores iguais ou acima do limite estabelecido pela referida resolução. A estação RC01 apresentou coeficiente de variação de $166 \%$ e a RC02 229,6\%, cujos valores foram influenciados principalmente pelos dados oriundos das datas de 09/12/03 e 05/01/04. Observa-se que na estação RC01 os maiores valores foram obtidos nos períodos de: 11 e 12/03; 04/04; 02 e 03/05 e 07/05.

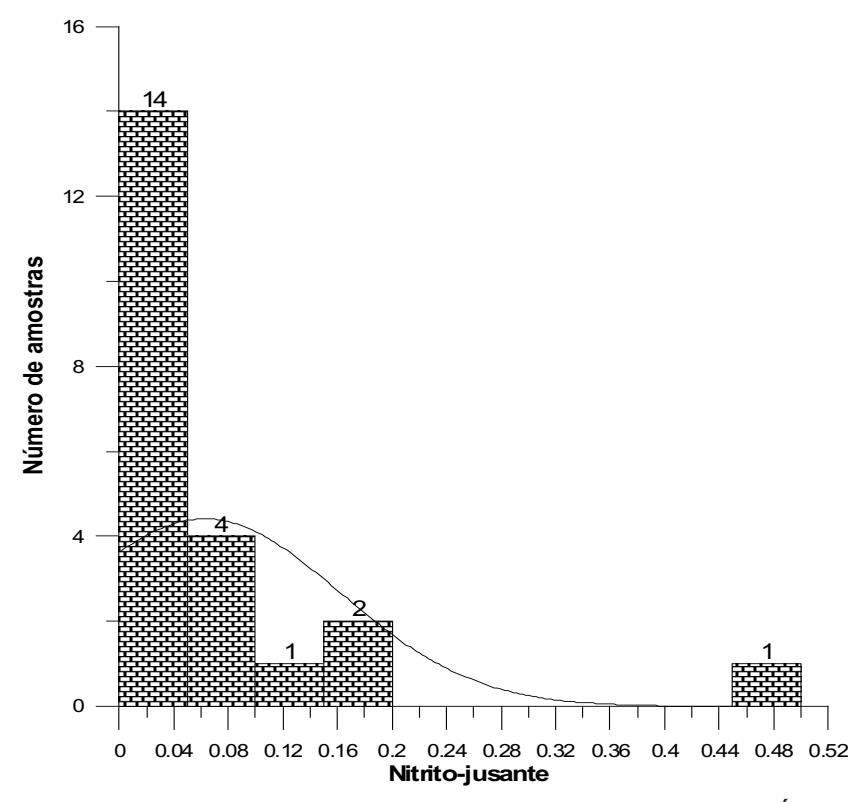

FIGURA 5 - HISTOGRAMAS DA VARIÁVEL N

Os valores do teor de nitrato cuja média a montante foi de $0,67 \mathrm{mg} / \mathrm{L}$ e a jusante de $0,78 \mathrm{mg} / \mathrm{L}$ de $\mathrm{N}$, não atingiram os limites constantes da Resolução $n^{\circ}$ 357/005 Conama (BRASIL, 2005), que é de $10 \mathrm{mg} / \mathrm{L}$ de N. A variabilidade dos dados a montante foi de $46,1 \%$ (Tabela 1) e a jusante de $60,1 \%$ (Tabela 2). Por outro lado em $59 \%$ das campanhas, os valores de nitrato foram maiores na estação de monitoramento RC02 e $31,8 \%$ na RC01. Como o nitrato geralmente está presente em concentrações moderadas nos corpos hídricos como metabólito natural do processo de nitrificação (conversão da amônia ou nitrito para o nitrato), valores elevados podem conduzir à proliferação em massa do fitoplanctôn e macrófitas, sobretudo em águas de baixa velocidade. Fatma (1999), British Columbia (1998) e Mc-
Em $72,7 \%$ dos resultados do conjunto de dados para as duas estações, a estação RC01 apresentou os maiores valores. Assim sendo pode-se inferir que provavelmente exista um processo de nitrificação no local, pois este composto normalmente aparece na água em uma fase intermediária natural do ciclo do Nitrogênio, na oxidação microbiana do nitrato. Normalmente o nitrito uma vez formado é imediatamente oxidado para a forma de nitrato (nitrificação) ou reduzido para a forma de Nitrogênio gás (desnitrificação). A distribuição dos dados (Figura 5) apresenta-se de forma assimétrica positiva, tanto a montante como a jusante.

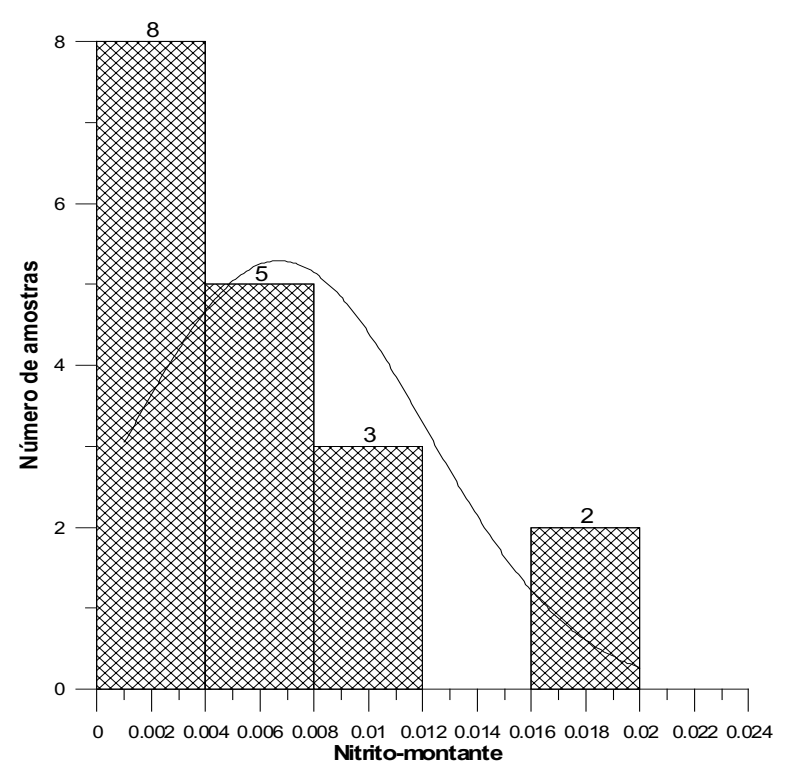

neely et al. (1979) informaram que as maiores fontes de aporte de nitrato são os adubos utilizados na fertilização do solo, efluentes domésticos industriais e dejetos de animais, não desconsiderando a fixação atmosférica. Os valores do nitrato referente às campanhas de 04/11/03; 09/12/04 e 30/05/05 são ligeiramente maiores do que os demais, influenciando na média e consequentemente no desvio padrão, cuja curva (Figura 6), e a distribuição dos dados apresentam-se de forma assimétrica positiva. Nas datas de 09/12/03; 05/01/04; 11/02/04 e 29/03/05, as concentrações estão acima da maioria do conjunto, influenciando no desvio padrão, consequentemente atuando diretamente na maior variabilidade dos dados em relação a montante, cuja curva de distribuição dos dados (Figura 6), tem forma assimétrica negativa. 

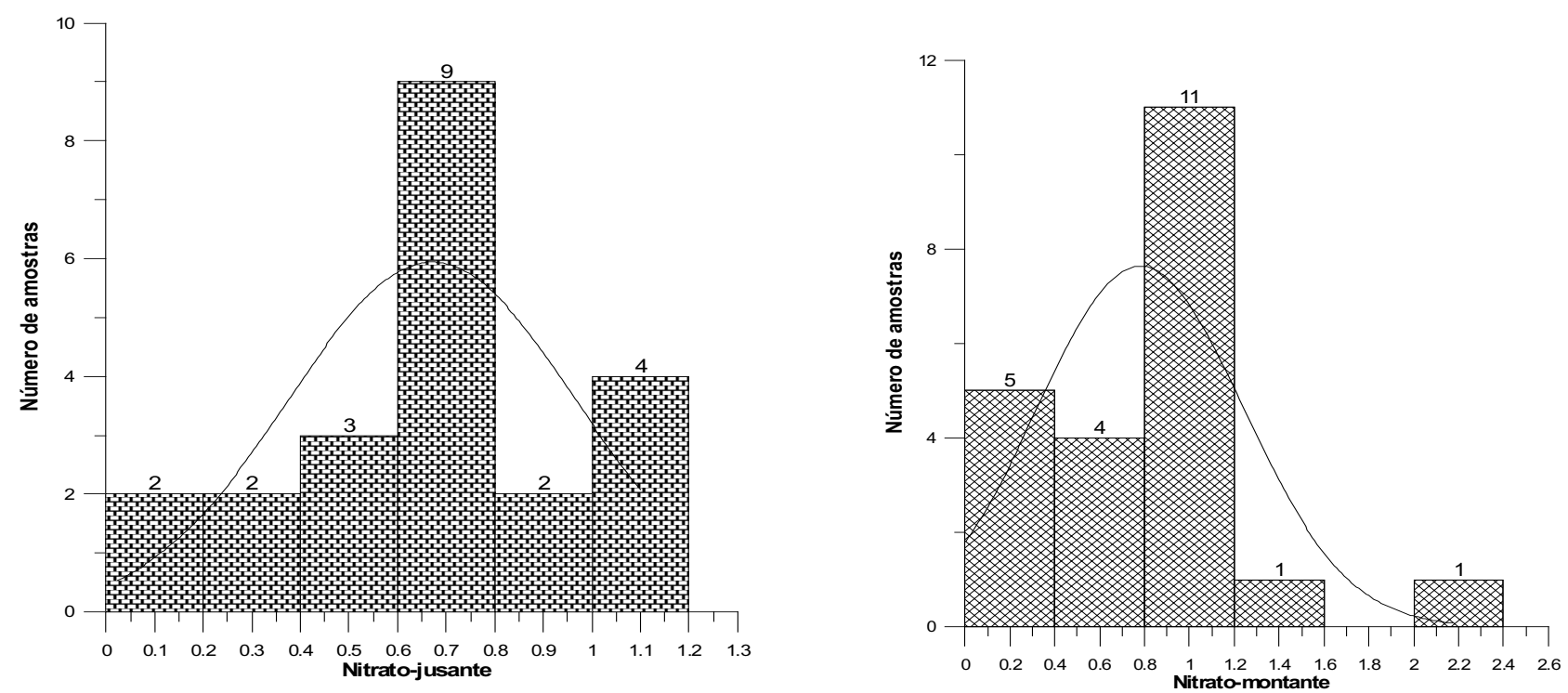

FIGURA 6 - HISTOGRAMAS DA VARIÁVEL NITRATO PARAAS ESTAÇÕES DE MONITORAMENTO RC01 E RC02

O nitrogênio amoniacal apresentou valores médios de 0,16 e 0,09 mg/L de $\mathrm{N}$ a montante e a jusante respectivamente, e que tais concentrações não se aproximam aos estabelecidos pela resolução do Conama $n^{\circ}$ 375/05 (BRASIL, 2005), cujo limite é de 3,7 $\mathrm{mg} / \mathrm{L}$ para valores de $\mathrm{pH}$ menor ou igual a 7,5. Alguns valores a montante e a jusante como aqueles obtidos nas campanhas de 09/12/03 e 05/01/04 estão acima dos demais, influenciando no coeficiente de variação $99 \%$ a montante e $156,4 \%$ a jusante (Tabelas 1 e 2 ), cujas curvas (Figura 7) apresentam assimetria positiva. Por outro lado a estação de monitoramento RC02

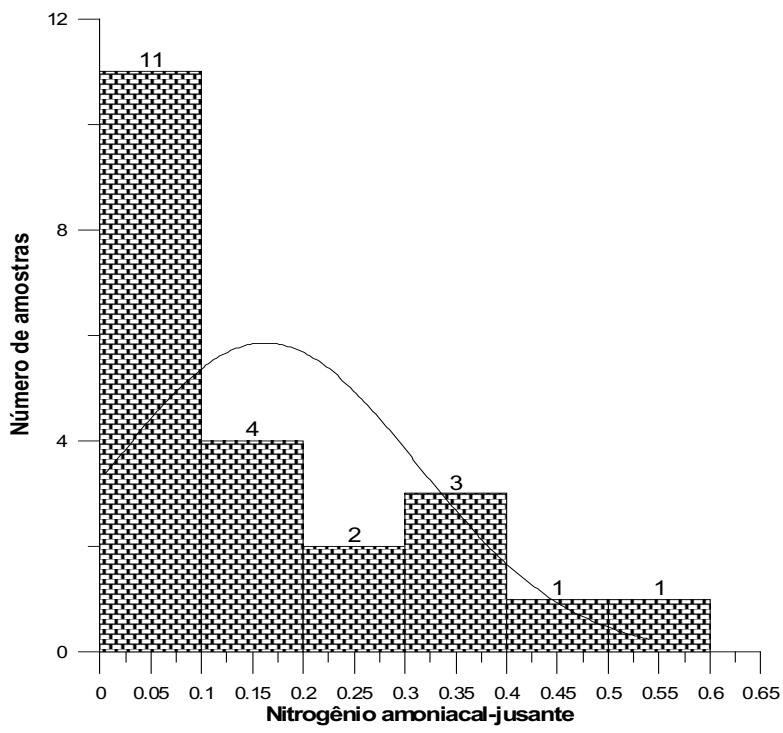

apresentou $54,5 \%$ dos valores maiores que a estação RC02. Como a amônia representa a forma primária do Nitrogênio, logo após a degradação dos compostos nitrogenados componentes das proteínas e aminoácidos, é possível que esta contaminação seja proveniente de fezes "in natura" ou de esgotos em fase de tratamento primário (NIEWEGLOSKY, 2006). Como o estudo refere-se a uma bacia hidrográfica rural, provavelmente exista a vinculação com a aplicação de dejetos suínos no solo, fezes de animais oriundos de pastagens ou no lançamento pontual no corpo hídrico.

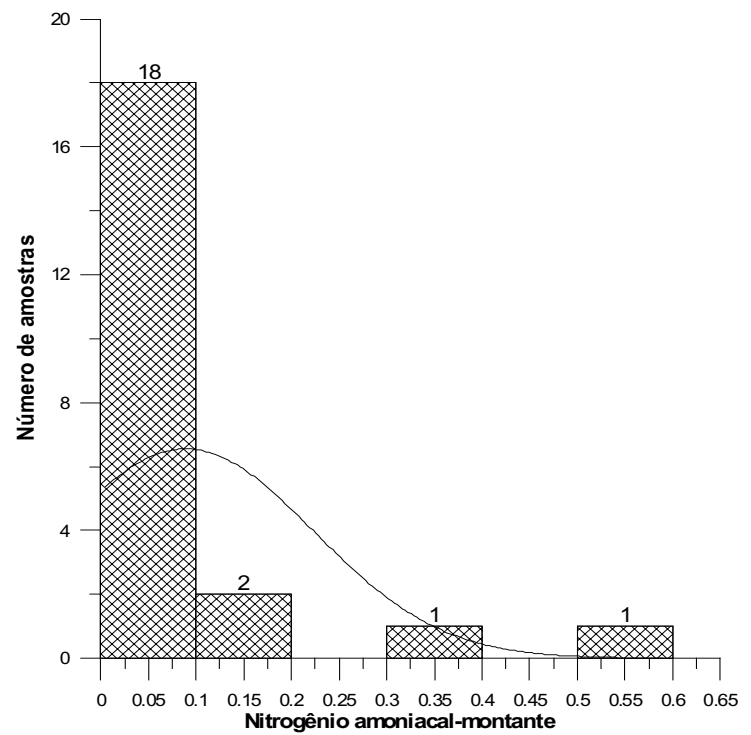

FIGURA 7 - HISTOGRAMAS DA VARIÁVEL NITROGÊNIO AMONIACAL PARAAS ESTAÇÕES DE MONITORAMENTO RC01 E RC02 
Com referência a variável Nitrogênio Total Kjeldahl (NTK), os teores médios foram de 0,48 e 0,33 $\mathrm{mg} / \mathrm{L}$ de $\mathrm{N}$ respectivamente para as estações RC01 e RC02, cuja resolução do Conama ${ }^{\circ}$ 375/05 (BRASIL, 2005), estabelece limite de $3,7 \mathrm{mg} / \mathrm{L}$. A estação RC01 apresentou uma variabilidade dos dados da ordem de

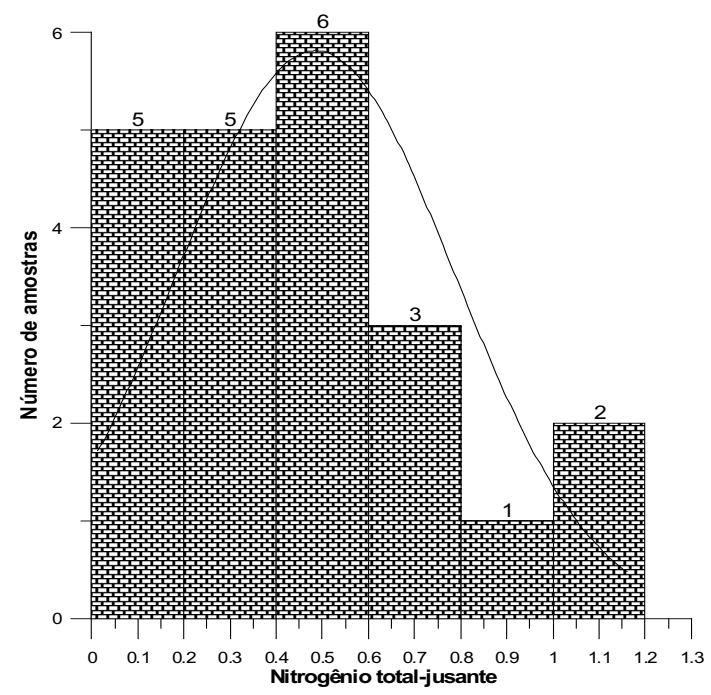

$63,6 \%$, enquanto que a RC02 a dispersão dos valores foi de $90,8 \%$ (Figura 8). Nos meses de fevereiro/2004, abril/2004, junho/2004, dezembro/2004, fevereiro/2005 e maio/20005, os valores do Nitrogênio Total Kjeldahl foram maiores do que o nitrato na estação RC01 e maior ou próximo na estação RC02.

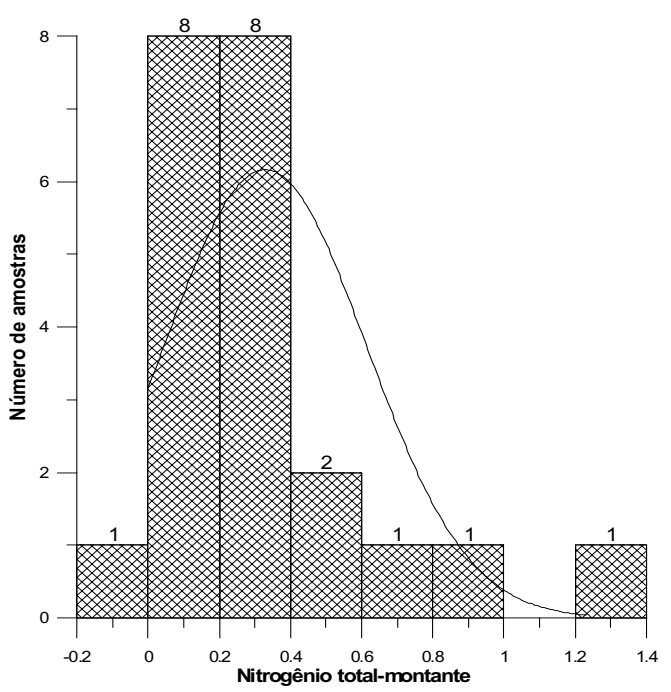

FIGURA 8 - HISTOGRAMAS DA VARIÁVEL NITROGÊNIO TOTAL KJELDHAL PARA AS ESTAÇÕES DE MONITORAMENTO RC01 E RC02

Observa-se que apenas a variável fósforo total (Tabela 5) apresentou diferença estatística significativa entre as duas estações (RC01 e RC02). Ainda na referida Tabela com exceção da variável DQO a qual ficou estável para as duas estações e a variável nitrato que aumentou a concentração na estação a jusante, as demais variáveis diminuíram sua concentração. Pode-

TABELA 5 - MÉDIAS SEGUIDAS POR LETRAS DIFERENTES NA LINHA SÃO ESTATISTICAMENTE DIFERENTES PELO TESTE T DE STUDENT

\begin{tabular}{ccc}
\hline VARIÁVEIS & MONTANTE & JUSANTE \\
\hline DBO5 & 5,19 & 4,32 \\
DQO & 11,04 & 11,16 \\
Fósforo Total & $0,13 \mathrm{a}$ & $0,07 \mathrm{~b}$ \\
Nitrato & 0,67 & 0,78 \\
Nitrito & 0,06 & 0,029 \\
Nitrogênio Amoniacal & 0,16 & 0,089 \\
Nitrogênio Kjehdal & 0,48 & 0,33 \\
\hline
\end{tabular}

se inferir sob o ponto de vista do conteúdo de matéria orgânica nos corpos hídricos, que não aconteceu o aporte via contribuição agrícola, através da fixação e ou adsorção destes nutrientes no solo. No entanto, a redução da concentração das variáveis para a estação a jusante pode ser explicada pela diluição no corpo hídrico em função da entrada de águas dos afluentes.

TABELA 6 - RELAÇÕES ENTRE: DBO ${ }_{5}$, DQO, NITROGÊNIO TOTAL KJELDHAL (NTK) E FÓSFORO TOTAL NAS ESTAÇÕES DE MONITORAMENTO DO RIO CONRADO

\begin{tabular}{cccc}
\hline RELAÇÃO & $\begin{array}{c}\text { EŚGOTOS } \\
\text { DOMÉSTICOS }\left(^{*}\right)\end{array}$ & RC01 & RC02 \\
\hline DBO5 : DQO & 0,6 a 0,8 & 0,47 & 0,4 \\
DBO5 : NTK & $17: 1$ & 11,25 & 13,33 \\
DQO : NTK & - & 23,75 & 33,63 \\
DBO5 : PTOTAL & $90: 1$ & 41,53 & 62,85 \\
DQO : PTOTAL & - & 87,69 & 158,57 \\
\hline NTK:PTOTAL & $6: 1$ & 3,69 & 4,71 \\
\hline$\left.{ }^{*}\right)$ Fonte: BRANCO (1978). & & &
\end{tabular}


Uma outra forma de analisar a questão é expressa na Tabela 6. O comportamento de um corpo hídrico com relação as variáveis que caracterizam a matéria orgânica analisada, pode ser feito através da relação DBO:DQO. Quando esta relação apresenta valores próximos a 0,5 indicaria o aporte de matéria orgânica oriundos de várias fontes (doméstica, comercial e industrial) e que potencialmente, contribuem tanto com compostos biodegradáveis como por orgânicos de difícil degradação biológica. No entanto para valores da ordem de 0,8 implicariam na existência de uma parcela preponderantemente biodegradável e, portanto, mais característico de corpos hídricos que recebem quase que exclusivamente esgotos domésticos. Analisando apenas o valor médio, a relação encontrada foi de 0,47 para a estação RC01 e de 0,4 para a RC02. Este fator indica que o rio Conrado recebe o aporte de matéria orgânica oriunda de várias fontes e a estação RC02 é mais influenciada pelas atividades agrícolas.

\section{CONCLUSÕES}

A estação de monitoramento RC01 apresentou como característica ter maiores concentrações dos nutrientes em função da atividade de criação de animais no divisor da bacia hidrográfica do rio Conrado; ao passo que a RC02 apresentou características do maior aporte da forma de nitrogênio oxidado.

Não foram observadas diferenças entre as duas estações de monitoramento que demonstrassem a contribuição mais consistente das práticas agrícolas desenvolvidas nesta bacia. As variáveis que caracterizam os compostos nitrogenados não apresentaram violações tendo-se por base o limite estabelecido pela Resolução n 375/05 do Conama (BRASIL, 2005). O fósforo total

\section{REFERÊNCIAS}

AGOSTINHO, A. A.; GOMES. Reservatório de Segredo: bases ecológicas para o manejo. Eduem: Maringá, 1997.

BRANCO, S. M. et al. Geoquímica Ambiental. Rubem Laina Porto. São Paulo: EDUSP. Associação Brasileira de Recursos Hídricos, 1991.

BRASIL. Resolução $n^{\circ}$. 357, de 17 de março de 2005. Dispõe sobre a classificação dos corpos de água e diretrizes ambientais para o seu enquadramento bem como estabelece
Por outro lado, observa-se uma maior quantidade de nitrogênio e fósforo do que carbono nas duas estações em função de que as relações $\mathrm{DBO}_{5}$ :NTK e DQO:P apresentaram-se abaixo dos valores para esgoto. A estação RC02 apresentou maior aproximação com os valores para o esgoto comparativamente a RC01. A relação NTK:P também apresentou-se abaixo daquela para o esgoto, sendo o maior valor novamente para a RC02. Nas práticas agrícolas onde se utiliza a rotação de culturas, a incorporação do nitrogênio e fósforo ocorre a partir das formas oxidadas (nitrato e fosfato), cujo nitrato não contribui para a concentração do NTK. Assim sendo, infere-se, por consequência, que o que estaria contribuindo para o aumento do NTK seria o nitrogênio na forma amoniacal e o fósforo típico de eliminação por dejetos de animais. Isso explicaria o fato de a relação $\mathrm{DBO}_{5}$ ser menor na estação RC01, face ao fato de que, no divisor de águas, a maior atividade é a criação de animais em áreas declivosas, favorecendo assim, o contato direto com o corpo hídrico.

apresentou $40 \%$ de violações com relação ao limite de 0,01 mg/L. ADQO mostrou um comportamento idêntico para as duas estações de monitoramento, e expôs que provavelmente não exista diferença no processo de oxidação das formas orgânicas e inorgânicas oxidáveis, para a condição de coletar amostras sem a interferência de cargas difusas.

Ocorreram violações de $27,3 \%$ e $13,7 \%$ respectivamente, para o conjunto de dados das estações de monitoramento RC01 e RC02 para a variável $\mathrm{DBO}_{5}$. Todas as variáveis apresentaram coeficiente de variação superior a $40 \%$. condições e padrões de lançamento de efluentes e dá outras providências. Disponível em: <http://www.mma.gov.br/port/ conama/> Acesso em: 15/09/2005

BRITISH COLUMBIA. Guidelines for interpreting Water Quality Data: Versão 1.0, 1998. Disponível em: <http://srmwww.gov. bc.ca/risc/pubs/aquatic/interp/interp-01.htm> Acesso em: 01/10/2006.

CANADÁ. Canadian Council of Ministers of the Environment. 
MACHADO, W. C. P. et al. Qualidade da água do Rio Conrado localizado na...

Canadian water quality guidelines for the protection aquatic life: Introduction. In: Canadian Environmental Quality Guidelines. Winnipeg, 1999. p.159.

CHAPMAN, D; KIMSTACH, V. Selection of water quality variables. In: CHAPMAN, D. Water quality assessments: a guide to the use of biota, sediments and water in environmental monitoring. 2 ed. Cambridge: UNESCO/WHO/UNEP, 1996.

COPEL. Companhia Paranaense de Energia Elétrica do Paraná. Estudos do Impacto Ambiental da Usina Hidroelétrica São João. Curitiba, 2001.

DEUTSCHER VERBAND FÜR WASSERWIRTSCHAFT UND KULTURBAUN - DVWK. Relevância de parâmetros de qualidade das águas aplicados à águas correntes. 1999.

ESTEVES, F. de A. Fundamentos de Limnologia. 2. ed. Rio de Janeiro: Interciência, 1998.

FATMA. Fundação do Meio Ambiente de Santa Catarina. Relevância de parâmetros de qualidade das águas aplicados a águas correntes. Parte I: Características gerais, nutrientes, elementos-traço e substâncias nocivas inorgânicas, características biológicas. Florianópolis: FATMA/GTZ, 1998, p.108.

MACHADO, W. C. P.; BECEGATO, V. A.; BITTENCOURT, A.V.L. Monitoramento ambiental da qualidade da água do rio Pinheiro - um afluente na bacia hidrográfica do rio Pato Branco-PR. Revista de Estudos Ambientais. v. 8 n. 2, p. 4463, 2006.
MANCUSO, P. C. S.; SANTOS, H. F. dos. (Eds.). Reúso da Água. Editora Manole, 2003.

McNEELY. R. N.; NEIMANIS, V. P.; DWYER. L. Water Quality Sourcebook. A Guide to Water Quality Parameters. Ottawa: Environment Canada, 1979. 90 p.

NIEWEGLOSWSKI, A. M. A. Indicadores de qualidade de água na bacia hidrográfica do rio Toledo - PR. Dissertação (Mestrado) - UFPR, Curitiba, 2006.

PARANÁ. SECRETARIA DE ESTADO DO MEIO AMBIENTE. Coletânea de legislação ambiental. Curitiba. GOVERNO DO ESTADO DO PARANÁ. 1996.

PEREZ, G. R. Fundamentos de Liminologia Neotropical. Medellín. Editora da Universidade de Antioquia, 1992.

SPERLING, M. Introdução à qualidade de água e tratamento de esgotos. DESA/EFMG, 1995.

TEIXEIRA, W. et al. Decifrando a terra. Ed. USP, 2000.

THORNTON, K. W. Perspectives on reservoir limnology. In: TRORNTON, K. W.; KIMMEL, B. L.; PAYNE, F. E. (Eds.). Reservoir Limnology: Ecological Perspectives. New York: John Wiley \& Sons, 1990.

WETZEL, R. G. Limnology. Phyladelphia: W.B. Saunders, 1983. 